\title{
THE EFFECT OF VERBAL FEEDBACK ON BIOMECHANICAL PERFORMANCE DURING SWIMMING ERGOMETRY
}

original paper

(1) University School of Physical Education in Wrocław

DOI: https://doi.org/10.5114/hm.2018.73606

\section{KRYSTYNA ZATOŃ, IZABELA CZEŚNIEWICZ, STEFAN SZCZEPAN}

Department of Swimming, University School of Physical Education in Wrocław, Wrocław, Poland

\begin{abstract}
Purpose. The aim was to investigate the effects of verbal feedback on biomechanical performance variables during swimming ergometry.

Methods. The total of 100 healthy and physically active males $(19.56 \pm 1.32$ years of age, $181.23 \pm 4.35 \mathrm{~cm}$ in height, and $70.54 \pm 8.6 \mathrm{~kg}$ in weight) participated in the study. The subjects were randomized into 2 groups (experimental and control); 2 sessions were administered. In the first session (pre-test), both groups completed the butterfly stroke on a swimming ergometer in identical conditions. In the second session (post-test), the experimental group received verbal instructions that conveyed kinaesthetic information. To rationalize verbal information, the criteria of efficient didactic communication were applied (syntax, semantics, and pragmatics). Biomechanical performance was monitored continuously by measuring work output $(\mathrm{J})$, total force $(\mathrm{N})$, power output $(\mathrm{W})$, and total distance of hand travel $(\mathrm{cm})$.
\end{abstract}

Results. Differences in the average values of the analysed variables between groups and samples were determined with a multivariate analysis of variance (MANOVA) $(p<0.05)$. The analysis of the results showed that the inclusion of verbal feedback resulted in statistically significant increases between pre- and post-test in all biomechanical variables in the experimental group, as well as in significant between-group differences post-test.

Conclusions. Appropriately prepared verbal cues following the criteria of efficient didactic communication may improve biomechanical performance during movement execution on a swimming ergometer.

Key words: swimming, verbal information, kinaesthetic information, work output, total force, power output

\section{Introduction}

The literature details a myriad of theoretical constructs explaining motor skill acquisition and later development. The classical definition is based on an information-processing model that involves separate stages between the stimulus and response and a feedback mechanism. It begins with the input of information from the environment through relevant sensory modalities. The sensory information is then processed by the central nervous system to determine the most appropriate action. The result is a response of observable motor activity [1].

It is recognized that effective motor performance requires feedback so as to allow the learner to improve movement execution. Typically, feedback is classified as intrinsic or extrinsic [2]. Intrinsic feedback is generally regarded as response produced and based on kinesthesis or proprioception. Proprioceptive feedback is related to information on the position of the body or a particular body part (joint position sense), whereas kinaesthetic feedback is the sense of movement and acceleration. They are interrelated and both involve several important neuromuscular sense organs: the vestibular apparatus provides the sense of balance and spatial orientation, muscle spindles detect changes in muscle length and velocity, while the Golgi tendon organs sense change in muscle tension [1]. This information is then used by the autonomous performer to augment pre-structured motor programs. Extrinsic feedback comes from an external source (e.g. stopwatch, verbal instructions from a teacher or coach, demon-

Correspondence address: Stefan Szczepan, Department of Swimming, University School of Physical Education in Wrocław, al. I.J. Paderewskiego 35, 51-612 Wrocław, Poland, e-mail: stefan.szczepan@awf.wroc.pl

Received: May 19, 2017

Accepted for publication: January 10, 2018

Citation: Zatoń K, Cześniewicz I, Szczepan S. The effect of verbal feedback on biomechanical performance during swimming ergometry. Hum Mov. 2018;19(1):3-9; doi: https://doi.org/10.5114/hm.2018.73606. 
stration, or video of the movement) and augments intrinsic feedback [3]. This type of feedback can be positive or negative and serves primarily to inform and motivate the learner. Extrinsic feedback is important as it reinforces correct movement execution (particularly by explaining movement phases) and improves performance [2, 4].

Besides the source, another important characteristic of feedback relates to its timing. Feedback can be concurrent (continuous information provided during the movement), immediate (in which discrete information is provided), or delayed (provided after some elapsed period of time) [1]. Behaviourist theories believe that feedback must be given immediately (and verbally) in order to reinforce correct execution $[5,6]$.

However, the delivery of information does not itself ensure a satisfactory outcome. The informative value of feedback is subject to numerous factors that may impede the transfer of knowledge, such as those related to the learning environment. For example, in the aquatic environment, perception is distorted, which makes the relay of intrinsic feedback difficult. This is a result of the physical properties of water (temperature, pressure, and density), as well as the forces exerted by water (buoyancy, drag). Hence, exteroceptive information may supplement the proprioceptive and kinaesthetic feedback loops.

Studies have mentioned that one way of improving movement execution is by modulating muscle tension, where improved control of muscle tension can enhance motor control and promote greater force production [7]. Furthermore, improved muscle tension can assist the muscle relaxation cycle when the muscle is no longer used [8]. Muscle tension also plays a large role in the kinaesthetic differentiation of movement, which governs movement precision and economy. It is treated as the ability to precisely determine the kinematic, spatial, and temporal properties needed in a movement while pairing these data with the previously stored motor programs [9]. Efforts aimed at improving the kinaesthetic differentiation of movement have been found to improve sensory processing and allow for more precise and efficient movement execution [10, 11].

Considering that verbal feedback has been found to be the most effective form of extrinsic feedback $[12,13]$, one can conclude that accordingly prepared verbal instruction can augment kinaesthetic information and improve motor performance. The result may be measured by changes in biomechanical variables quantifying the effectiveness and efficacy of a given movement.

The swim training programme contains sessions that involve the development of strength and endurance.
Mostly, they are executed on a swimming ergometer and have a positive influence on the kinematic parameters of the swim stroke [14, 15]. Extension of swim stroke and optimization of stroke rate lead to progression of achievements and results [16]. Therefore, it is important that dry land training which includes swimming with an ergometer is optimized. One method for improving swimming ergometry performance could be a precise development of suitable verbal feedback transferring knowledge on kinaesthetic sensation during swim ergometry.

\section{Study aim and hypothesis}

The purpose of this study was to determine the effects of verbal feedback on biomechanical variables during swimming ergometry. It was hypothesized that verbal cues transmitting kinaesthetic information could improve swimming ergometry performance.

\section{Material and methods}

\section{Participants}

The total of 100 healthy and physically active males were recruited (age: $19.56 \pm 1.32$ years; height: 181.23 $\pm 4.35 \mathrm{~cm}$; mass: $70.54 \pm 8.6 \mathrm{~kg}$ ). All were untrained with a similar fitness profile as ascertained via a questionnaire. The participants were randomly assigned to an experimental or control group.

\section{Procedure}

Informed consent was obtained from all participants and the research protocol was approved by the local ethics committee. All procedures were performed at the same time of day in an indoor facility with controlled environmental conditions (temperature, humidity, and air movement).

The participants were to perform an arm movement mimicking the butterfly stroke constant on a swim ergometer (Weba Sport und Med. Artikel GmbH, Germany). The apparatus consisted of a bench with two independent handles and a computer that measured the total work output $(\mathrm{J})$, total force $(\mathrm{N})$, total power (W), total distance of hand travel (cm). After the trials, all of the data were sent on for further analysis. A swim metronome was used to maintain pace (one stroke per $2.5 \mathrm{~s}$ ), where the return to the initial position with the arms stretched over the head and in front of the participant was treated as one stroke cycle. Two sessions were administered. The first session (pre-test) was per- 
formed by both groups with no verbal feedback provided. In order to minimize the effects of fatigue, the second session (post-test) was performed after a period of 48 hours, during which the experimental group received verbal feedback from one of the study authors.

\section{Measurement}

The test duration was $10 \mathrm{~min}$, with data recorded during the first 5 min while the remaining 5 min served as a cool-down. Biomechanical variables that were recorded in the 5-min test window included:

1. the total work output $(\mathrm{J})$ summed across test duration;

2. the total force $(\mathrm{N})$ as the sum of the force exerted on the handles;

3. total power $(\mathrm{W})$;

4. total distance of hand travel $(\mathrm{cm})$ as the displacement of the hand.

\section{Verbal feedback}

A pilot study was conducted to design and verify the intervention protocol in the experimental group. The purpose was to develop suitable verbal feedback transferring knowledge on kinaesthetic sensation during swim ergometry. This portion of the study involved 20 males not recruited in the experiment proper. Two sessions were administered. In the first session, no feedback was delivered except for the information if the stroke movement was being correctly performed. After a 14-day interval, the second session was held, in which verbal feedback was successively introduced.

To rationalize verbal information, the criteria of efficient didactic communication were applied (syntax, semantics, and pragmatics). In this context, syntax is a classification of motor activities into sequences of movement. This means that the transmitted information should be ordered and reflect the motions performed in sequence. Semantics is a uniform expression for a given movement shared by the trainer and swimmer. In practice, it is necessary to establish the shared scope of information in order for it to be comprehensible for the person performing the movement. Pragmatics, i.e. utility, refers to the physical capacities of the participant, that is, accounting in verbal messages for the motor capacities of the swimmer [5, 8].

In addition, the structure of the human memory was also considered, and it was agreed that it can only store a limited volume of information [17, 18]. Therefore, it was necessary to eliminate any superfluous data which might have been transmitted to the swimmer in order to preserve the short-term memory potential. In this manner, the verbal messages were developed.

The protocol was designed to convey information on how to execute the motor task with minimal physiological cost. The information consisted of the following statements:

1. Lay on the bench so that no tension is felt in any part of the body.

2. Comfortably hold onto the handles so that the fingertips touch the edge.

3. Position the arms with slightly bent elbows.

4. Slightly flex the hands.

5. Swim with the arms at a constant pace.

6. Squeeze the handles using the entire surface of the hand.

7. Perform smooth movements and do not jerk the arms.

8. Tense the muscles when pulling on the handles but relax during the recovery.

9. Tense the buttock and back muscles when pulling with the arms.

10. Pull the arms until they reach the hip.

Information 1-10 was used during the test. Each mistake was corrected by way of ongoing feedback.

\section{Statistical analysis}

Basic descriptive statistics (mean and standard deviation) were calculated for all biomechanical variables. To compensate for learning effects, the mean values of all data obtained in the post-test were increased by the baseline values (pre-test) in both the control and experimental groups. Differences in the average values of the analysed variables between groups and samples were determined with multivariate analysis of variance (MANOVA) [2]. Levene's test was applied to confirm the homogeneity of variance. Duncan's test was applied for post-hoc comparisons. The significance level for all statistical procedures was set at $p<0.05$.

\section{Ethical approval}

The research related to human use has been complied with all the relevant national regulations and institutional policies, has followed the tenets of the Declaration of Helsinki, and has been approved by the authors' institutional review board or an equivalent committee.

\section{Informed consent}

Informed consent has been obtained from all individuals included in this study. 


\section{Results}

Table 1 presents the performance characteristics attained at pre- and post-test for both groups. The mean total force per stroke increased significantly by $\Delta 18.49 \mathrm{~N}$ in the experimental group and by $\Delta 1.7 \mathrm{~N}$ in the control group (Figure 1). The mean work output per stroke rose by $\Delta 19.04 \mathrm{~J}$ in the experimental group and by $\Delta 0.79 \mathrm{~J}$ in the control group, with the former increase statistically significant (Figure 2). The mean power per stroke increased significantly by $\Delta 7.93 \mathrm{~W}$ in the experimental group and by $\Delta 0.72 \mathrm{~W}$ in the control group (Figure 3). The mean distance of hand travel per stroke increased significantly by $\Delta 16.97 \mathrm{~cm}$ in the experimental group, and declined by $\Delta-0.33 \mathrm{~cm}$ in the control group (Figure 4).

The analysis of the results showed that the inclusion of verbal feedback resulted in statistically significant increases in the experimental group between preand post-test in all biomechanical variables, as well as significant between-group differences at post-test (Table 1).

\section{Discussion}

The classical theory of motor learning and control [19] holds that feedback (intrinsic or extrinsic) can determine movement execution and performance. Many authors have studied the effects of information feedback, including efforts at optimizing swimming technique $[4,5,20]$. It has been documented over many years that feedback plays a significant role in progressively developing motor control. However, the literature is limited on the relationship between verbal feedback and movement effectiveness, signalling the need for additional research in this field.

The few existing studies on the use of feedback to improve motor function have focused on the use of verbal instruction to modify motor behaviour [12, 21, 22]. These studies investigated the impact of feedback in

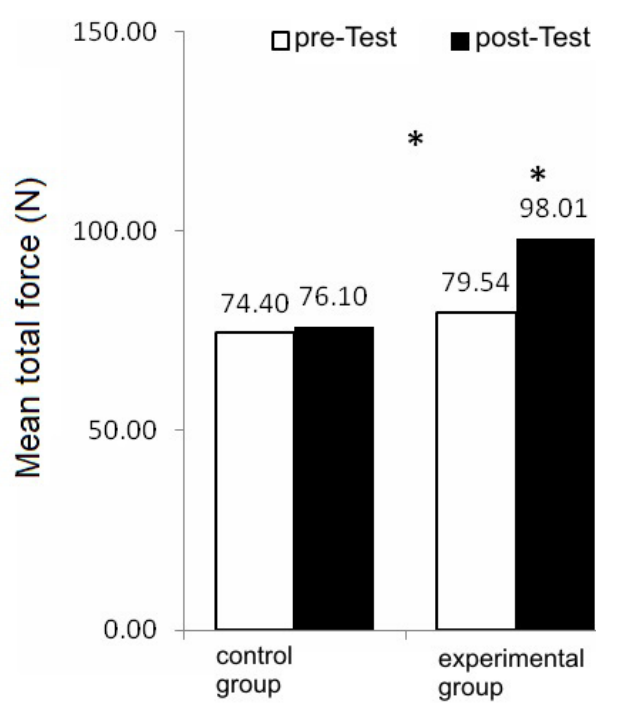

* statistically significant difference in the experimental group between preand post-test, as well as between-group difference at post-test at $p<0.05$

Figure 1. Pre- and post-test mean total force per stroke for both groups

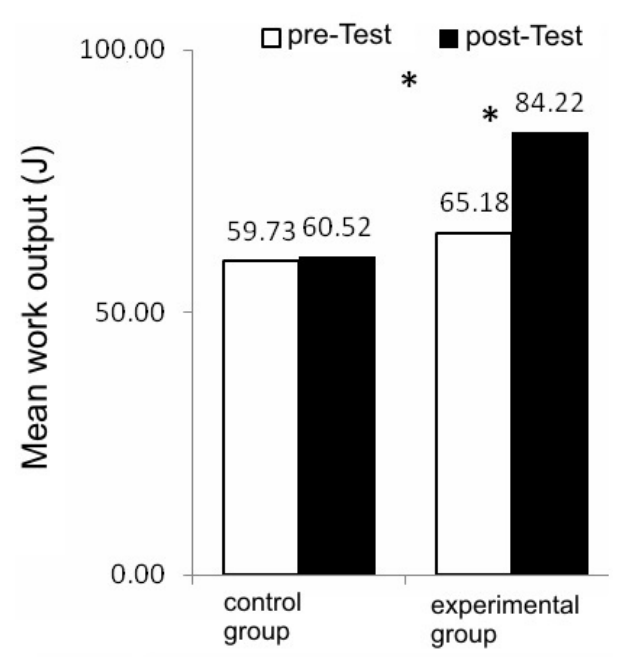

* statistically significant difference in the experimental group between preand post-test, as well as between-group difference at post-test at $p<0.05$

Figure 2. Pre- and post-test mean work output per stroke for both groups

Table 1. Pre- and post-test swim ergometer performance for both groups

\begin{tabular}{lcccccc}
\hline \multirow{2}{*}{ Biomechanical measures } & \multicolumn{3}{c}{ Control group } & & \multicolumn{3}{c}{ Experimental group } \\
\cline { 2 - 7 } & pre-test & post-test & $\Delta$ & pre-test & post-test & $\Delta$ \\
\hline Mean total force $(\mathrm{N})$ & $74.40 \pm 14.10$ & $76.10 \pm 13.20$ & 1.70 & $79.54 \pm 19.50$ & $98.01 \pm 20.90^{*}$ & 18.49 \\
Mean work output $(\mathrm{J})$ & $59.73 \pm 15.70$ & $60.52 \pm 15.90$ & 0.79 & $65.18 \pm 23.80$ & $84.22 \pm 17.80^{*}$ & 19.04 \\
Power output $(\mathrm{W})$ & $24.43 \pm 8.10$ & $25.15 \pm 7.00$ & 0.72 & $27.5 \pm 10.70$ & $35.43 \pm 9.40^{*}$ & 7.93 \\
Distance of hand travel $(\mathrm{cm})$ & $174.21 \pm 22.70$ & $173.88 \pm 23.20$ & -0.33 & $173.82 \pm 27.90$ & $190.79 \pm 16.40^{*}$ & 16.97 \\
\hline
\end{tabular}

* statistically significant difference in the experimental group between pre- and post-test, as well as significant betweengroup difference at post-test at $p<0.05$ 


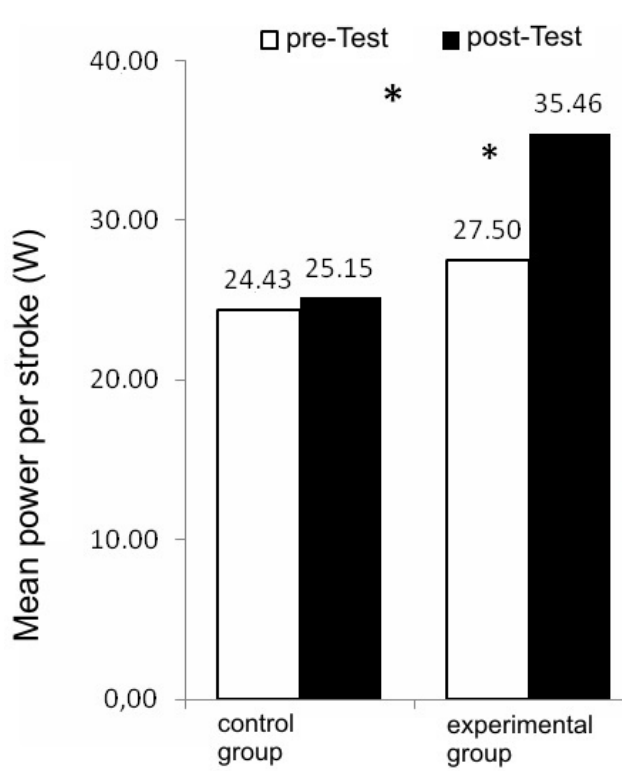

* statistically significant difference in the experimental group between preand post-test, as well as between-group difference at post-test at $p<0.05$

Figure 3. Pre- and post-test mean power per stroke for both groups

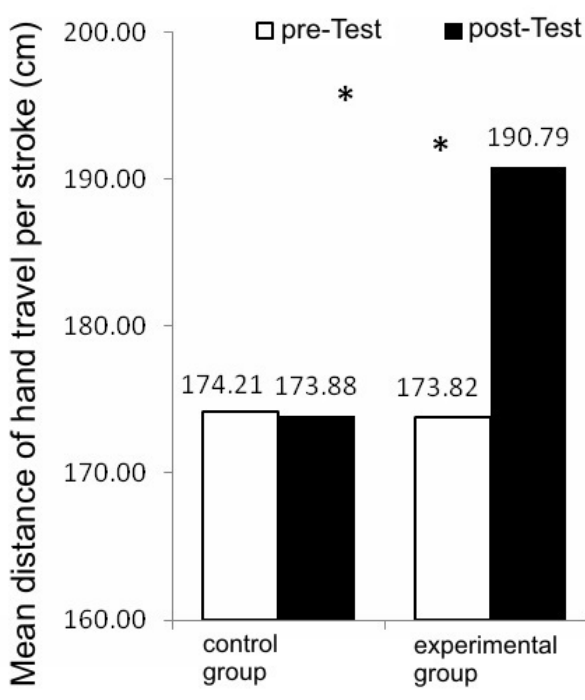

* statistically significant difference in the experimental group between preand post-test, as well as between-group difference at post-test at $p<0.05$

Figure 4. Pre- and post-test mean distance of hand travel per stroke for both groups

activities of daily living, as well as sport-specific motor tasks, including soccer [23] and tennis [24]. Other experiments concentrated on the effects of the quantity, frequency, and form of information needed to facilitate motor skill acquisition [25, 26] or the effects of time on retention following verbal feedback [5]. In all of the aforementioned studies, verbal information had a beneficial effect on task effectiveness and performance, suggesting that augmented feedback is an important element in motor learning and development. Many investigators have concluded that verbal feedback is the most effective form of information delivery [12].
While kinaesthetic or proprioceptive feedback does relay critical information, it may be insufficient in generating efficient motor programs, particularly when the movement is performed in an alternative environment. Therefore, verbal feedback conveying kinaesthetic information can provide additional, complementary knowledge on joint position, muscle force production, or body position. Furthermore, information orally transmitted to a learner needs to follow certain pre-established criteria for effective communication, including semantics, pragmatics, and syntax [5, 8]. Considering the above requirements, we introduced a series of clear and concise instructions with a kinaesthetic component and hypothesized that such verbal feedback could improve performance in swim ergometry.

This was confirmed, as the studied biomechanical measures increased significantly in the verbal feedback condition. This suggests that verbal feedback following coherent didactic protocols can improve biomechanical performance and can have profound practical implications, particularly when a motor task is performed in an aquatic environment.

Coaches agree that swimming training should include sessions both on land and in water. The achievement of top performance in swimming is not possible without dry land exercises, e.g. strength and conditioning training on a swimming ergometer [14]. During the training cycle, swimmers train on land approximately 300 hours per year. The time devoted to developing maximum strength and condition constitutes $60 \%$ of the total working time on land [15]. Dry land strength training improves the swimming performance, as reflected in related parameters, such as increased stroke length [16]. Therefore, it seems important to optimize performance while swimming with ergometer by providing rationalized verbal feedback regarding kinaesthetic information, which modifies motor behaviour and leads to progression of achievements.

When composing feedback, it is important to take into account criteria for efficient instructional communication. Verbal messages should be ordered, and reflect movements performed in sequence (syntax). In addition, the trainer and swimmer should establish a uniform scope of understandable information (semantics). This information should refer to the physical capacities of the swimmer, and not go beyond the swimmer's motor skills (pragmatics) [5, 8].

Carefully prepared verbal feedback conveying kinaesthetic information can influence the values of produced power or force by correcting stroke flaws. In effect, training goals can be reached faster and be more precise. 


\section{Conclusions}

Verbal feedback conveying kinaesthetic information modifies motor behaviour. This was observed by modification of the biomechanical variables under study following verbal feedback, which suggests that appropriately prepared verbal cues fulfilling the criteria of efficient didactic communication, such as those related to syntax, semantics and pragmatics, can enhance work output, total force, power output, and distance of hand travel during movement execution. A direct implementation of the results of this study to the practice of coaching and teaching would increase the effectiveness of training methods, as well as improve the performance of movement during practice.

\section{Disclosure statement}

No author has any financial interest or received any financial benefit from this research.

\section{Conflict of interest}

The authors state no conflict of interest.

\section{References}

1. Schmidt RA, Lee TD. Motor control and learning. A behavioral emphasis, 5th edition. Champaign: Human Kinetics; 2011.

2. Wrisberg C. Sport skill instruction for coaches. Champaign: Human Kinetics; 2007.

3. Proteau L, Carnahan H. What causes specificity of practice in a manual aiming movement: vision dominance or transformation errors? J MotBehav. 2001;33(3):226234; doi: 10.1080/00222890109601908.

4. Silverman S, Woods AM, Subramaniam PR. Task structures, feedback to individual students, and student skill level in physical education. Res Q Exerc Sport. 1998; 69(4):420-424; doi: 10.1080/02701367.1998.10607718.

5. Zatoń K, Szczepan S. The impact of immediate verbal feedback on the improvement of swimming technique. J Hum Kinet. 2014;41(1):143-154; doi: 10.2478/ hukin-2014-0042.

6. Lee TD, Swinnen SP, Serrien DJ. Cognitive effort and motor learning. Quest. 1994;46(3):328-344; doi: 10.1080/ 00336297.1994.10484130.

7. Proske U, Gandevia SC. The kinaesthetic senses. J Physiol. 2009;587(Pt 17):4139-4146; doi: 10.1113/jphysiol.2009.175372.

8. Zatoń K. The role of verbal information about sensory experience from movement apparatus in the process of swimming economization. In: Kjendlie PL, Stallman RK, Cabri J (eds.). Biomechanics and medicine in swimming XI: proceedings of the $\mathrm{XI}^{\text {th }}$ International Symposium for Biomechanics and Medicine in Swimming. Oslo: Norwegian School of Sport Sciences; 2010; 349-351.
9. Lovelace EA, Aikens JE. Vision, kinesthesis, and control of hand movement by young and old adults. Percept Mot Skills. 1990;70(3 Pt 2):1131-1137; doi: 10.2466/ pms.1990.70.3c.1131.

10. Fung YC. Biomechanics: motion, flow, stress, and growth. New York: Springer; 1990.

11. Klarowicz A, Zatoń K, Albiński P. Differences in conscious reception of stimuli from water environment in school children participating in rehabilitation swimming program. Pol J Environ Stud. 2006;15(5B):643-645.

12. Landin D. The role of verbal cues in skill learning. Quest. 1994;46(3):299-313; doi: 10.1080/00336297. 1994.10484128.

13. More KG, Franks IM. Analysis and modification of verbal coaching behaviour: the usefulness of a data-driven intervention strategy. J Sports Sci. 1996;14(6):523-543; doi: 10.1080/02640419608727739.

14. Tanaka H, Costill DL, Thomas R, Fink WJ, Widrick JJ. Dry-land resistance training for competitive swimming. Med Sci Sports Exerc. 1993;25(8):952-959; doi: 10.1249/00005768-199308000-00011.

15. Sadowski J, Mastalerz A, Gromisz W, Jówko E, Buszta M. The effects of swimming and dry-land resistance training programme on non-swimmers. Pol J Sport Tourism. 2015;22:35-40; doi: 10.1515/pjst-2015-0011.

16. Girold S, Maurin D, Dugué B, Chatard JC, Millet G. Effects of dry-land vs. resisted- and assisted-sprint exercises on swimming sprint performances. J Strength Cond Res. 2007;21(2):599-605; doi: 10.1519/R-19695.1.

17. Marteniuk RG. Information processing in motor skills. New York: Holt, Rinehart and Winston; 1976.

18. Miller GA. The magical number seven plus or minus two: some limits on our capacity for processing information. Psychol Rev. 1956;63(2):81-97; doi: 10.1037/h0043158.

19. Adams JA. A closed-loop theory of motor learning. J Mot Behav. 1971;3(2):111-149; doi: 10.1080/00222895. 1971.10734898.

20. Magill RA. The influence of augmented feedback on skill learning depends on characteristics of the skill and the learner. Quest. 1994;46(3):314-327; doi: 10.1080/ 00336297.1994.10484129.

21. Kernodle MW, Johnson R, Arnold DR. Verbal instruction for correcting errors versus such instructions plus videotape replay on learning the overhead throw. Percept Mot Skills. 2001;92(3 Pt 2):1039-1051; doi: 10.2466/ pms.2001.92.3c.1039.

22. Laguna PL. Task complexity and sources of task-related information during the observational learning process. J Sports Sci. 2008;26(10):1097-1113; doi: 10.1080/02640410801956569.

23. Franks IM. The use of feedback by coaches and players. In: Reilly T, Bangsbo J, Hughes M (eds.). Science and football III. London: E \& FN Spon; 1997; 267-278.

24. Hebert EP, Landin D. Effects of a learning model and augmented feedback on tennis skill acquisition. Res Q Exerc Sport. 1994;65(3):250-257; doi: 10.1080/02701367. 1994.10607626. 
25. Boyce BA. The effects of an instructional strategy with two schedules of augmented KP feedback upon skill acquisition of a selected shooting task. J Teach Phys Educ. 1992;11(1):47-58; doi: 10.1123/jtpe.11.1.47.

26. Wulf G, Shea CH. Principles derived from the study of simple skills do not generalize to complex skill learning. Psychon Bull Rev. 2002;9(2):185-211; doi: 10.3758/ BF03196276. 